\title{
Diagnóstico dos Indicadores de Consumo de Água em Escolas Públicas de Recife-PE
}

Analysis of Water Consumption Indicators in Public Schools of Recife-PE

Diagnóstico de Indicadores de Consumo de Agua en las Escuelas Públicas de Recife-PE

Anna Elis Paz Soares

Estudante de Engenharia Civil, UPE, Brasil.

anna_elispaz@hotmail.com

Luiz Gustavo Costa Ferreira Nunes

Estudante de Engenharia Civil, UPE, Brasil.

I.gustavo.nunes@hotmail.com

Simone Rosa da Silva

Professor Doutora, UPE, Brasil.

simonerosa@poli.br 


\section{RESUMO}

A escola deve ter papel de destaque na formação de cidadãos conscientes quanto ao uso sustentável da água. No entanto, o ambiente escolar é classificado por diversos autores como um forte consumidor e desperdiçador de água, principalmente em se tratando de escolas públicas. Inserido nesse contexto, o presente trabalho contém estudos realizados em duas escolas públicas estaduais da cidade de Recife-PE, objetivando o diagnóstico do padrão de consumo dessa tipologia predial. A metodologia empregada consistiu no monitoramento setorizado do consumo de água a partir da instalação de hidrômetros nos principais pontos de uso desse recurso, por um período de um ano, além do cálculo do Indicador de Consumo (litros/aluno*dia). Os resultados mostraram que os setores de uso exclusivo da comunidade docente e funcionários de uma forma geral são os maiores consumidores de água. Em contrapartida, as duas escolas apresentaram um baixo Indicador de Consumo em comparação com outros estudos realizados em outras instituições de ensino no Brasil e no mundo. A análise desses indicadores deve orientar o desenvolvimento de atividades para sensibilização dos usuários direcionadas para cada setor específico, contribuindo para a redução do desperdício de água.

PALAVRAS-CHAVE: Indicador de Consumo. Escolas Públicas. Consumo de Água.

\section{ABSTRACT}

The school should have a prominent role in the formation of citizens aware about the sustainable use of water. However, the school environment is classified by several authors as a strong water consumer and waster, especially when it comes to public schools. Inserted in this context, this work contains studies conducted in two public schools in the city of Recife-PE, aiming to identify the characteristics of water use of this building typology. The methodology consisted of divide the schools in sectors to study the water consumption of each sector through the installation of water meters in specific locations, for a period of one year, in addition to calculating the Consumption Indicator (liters / student* day). The results showed that sectors involving workers and teaching staff in general are the largest consumers of water. In contrast, the two schools had a low consumption indicator compared to other studies in other educational institutions in Brazil and worldwide. The analysis of these indicators should guide the development of activities to raise awareness of users directed to each specific sector, contributing to the reduction of water waste.

KEYWORDS: Consumption Indicator. Public schools. Water consumption.

\section{RESUMEN}

La escuela debe tener un papel destacado en la formación de ciudadanos conscientes sobre el uso sostenible del agua. Sin embargo, el entorno escolar se clasifica por varios autores como un fuerte consumo y desperdicio de agua, específicamente cuando se trata de escuelas públicas. Insertado en este contexto, este trabajo contiene estudios llevados a cabo en dos escuelas públicas en la ciudad de Recife-PE, con el objetivo de diagnosticar el patrón de consumo de agua desta tipología de constructión. La metodología consistió en el monitoreo sectorial del consumo de agua a través de la instalación de contadores de agua en los principales puntos de la utilización de este servicio por un periodo de un año, además de calcular el indicador de consumo (litros/alumno*día). Los resultados mostraron que el uso exclusivo de los sectores de la comunidad y el personal docente en general, son los mayores consumidores de agua. En contraste, las dos escuelas presentaron un indicador de bajo consumo en comparación con otros estudios en otras instituciones educativas en Brasil y en todo el mundo. El análisis de estos indicadores debe guiar el desarrollo de las actividades de sensibilización de los usuarios dirigidas a cada sector específico, lo que contribuye a la reducción del desperdicio de agua.

PALAVRAS CLAVE: Indicador de Consumo. Las Escuelas Públicas. Consumo de Agua. 


\section{INTRODUÇÃO}

O Brasil, em termos globais, apresenta uma situação confortável quanto aos recursos hídricos. Representa $53 \%$ da produção de água do continente sul americano e $12 \%$ do total mundial. No entanto, apesar desse aparente conforto, existe uma distribuição espacial desigual dos recursos hídricos no território brasileiro. Cerca de $80 \%$ da produção hídrica brasileira encontrase na região hidrográfica Amazônica, onde se encontra o menor contingente populacional e valores reduzidos de demandas consuntivas (ANA, 2013).

Além da má distribuição geográfica dos recursos hídricos, há a escassez crescente de mananciais com qualidade e quantidade suficiente para abastecer os grandes centros urbanos. Visto que nas bacias hidrográficas com maiores índices de urbanização e industrialização, o uso e a poluição dos recursos hídricos são normalmente mais intensos. A consequência desse quadro é a elevação exponencial dos custos com captação, adução e tratamento da água bruta (PNCDA, 2003).

O desperdício é outro fator limitante do acesso à agua potável. Cheung et al (2009) pontuam que o termo desperdício compreende as perdas evitáveis, que correspondem à negligência do usuário que não tem consciência ambiental. Segundo Rebouças (1997), ele deriva tanto da baixa eficiência dos serviços de saneamento básico, situação caracterizada pelas grandes perdas de água tratada nas redes de distribuição (entre $25 \%$ e $60 \%$ ), quanto dos grandes desperdícios gerados pela cultura da abundância e pelo obsoletismo dos equipamentos hidrossanitários. Rebouças (2001) defende que é possível racionalizar o uso da água seguindo o exemplo dos programas para uso racional da água (PURA) desenvolvidos em algumas instituições de ensino no Brasil e em outros países mais desenvolvidos, para tanto, faz-se necessário estimular a substituição de equipamentos obsoletos por outros mais eficientes e desenvolver campanhas permanentes de informação à população em geral, incluindo ensinamentos de como usar a água disponível nos programas das escolas de 1 으 e 20 graus.

A escola é um dos ambientes mais importantes para a transformação das crianças e jovens em cidadãos. Por isso, é imprescindível a abordagem de temas relacionados à agua, não só aos seus aspectos físicos, químicos e biológicos, como também aos métodos para utilizá-la de forma sustentável. No entanto, a escola é identificada por diversos autores como um forte consumidor e desperdiçador de água. Melo et al (2014) pontuam que o desperdício de água devido às perdas por vazamentos ou pelo mau uso desse insumo é de grande magnitude em escolas públicas. Gonçalves et al (2005) apontam como causas para essa realidade a falta de sensibilização dos usuários com relação à conservação do ambiente, a não responsabilidade direta pelo pagamento da conta de água, e a inexistência ou ineficiência de um sistema de manutenção.

Melo et al (2014) acrescentam que o consumo de água é influenciado pelo nível de esclarecimento e conhecimento dos usuários sobre a importância da conservação dos recursos naturais, da maior ou menor disponibilidade hídrica do local, da existência ou não de sistema de medição do volume consumido, dos preços pagos pela água, entre outros aspectos de natureza sociocultural, regional e econômica. 
Sendo assim, prioritariamente ao desenvolvimento de ações para redução do consumo de água nas edificações escolares, deve ser realizado um diagnóstico preliminar da situação da edificação, visando à identificação do padrão de consumo dessa tipologia predial.

O Indicador de Consumo (IC), relação entre o volume de água consumido por agente consumidor em um determinado período de tempo, pode ser utilizado como um valor de referência para determinar se um sistema pode ser melhorado. O Quadro 1 resume valores de IC encontrados em diversos estudos no Brasil e em outros países. Como pode ser observado, os valores variam de 4,5 L/(agente consumidor.dia) até $134 \mathrm{~L} /($ agente consumidor.dia).

Quadro 1: Indicadores de Consumo em diferentes localidades

\begin{tabular}{|c|c|c|}
\hline Referência & Região & $\begin{array}{l}\text { Indicador de Consumo } \\
\text { (L/aluno/dia) }\end{array}$ \\
\hline Farina et al, 2011 & Bolonha, Itália & 10 a 30 \\
\hline Cheng et al, 2004 & Taiwan & 34 \\
\hline Gonçalves et al, 2005 & Campinas, SP & 51,1 \\
\hline $\begin{array}{c}\text { AQUA, } 2007 \\
\text { (após implantação do PURA) }\end{array}$ & São Paulo & 10 a 20 \\
\hline $\begin{array}{c}\text { AQUA, } 2007 \\
\text { (após implantação do PURA) }\end{array}$ & França & 13 \\
\hline Oliveira, 2013 & Ituiutaba, MG & 13,86 a 51,26 \\
\hline Oliveira, 2013 & Monte Carmelo, MG & 10,59 a 62,23 \\
\hline Oliveira, 2013 & Patos de Minas, MG & 6,42 a 40,21 \\
\hline Oliveira, 2013 & Patrocínio, MG & 7,15 a 20,61 \\
\hline Oliveira, 2013 & Uberaba, MG & 8,26 a 62,82 \\
\hline Oliveira, 2013 & Uberlândia, MG & 7,27 a 47,4 \\
\hline Oliviera, 1999 & Campinas, SP & 81,1 \\
\hline $\begin{array}{c}\text { Oliviera, } 1999 \\
\text { (após implantação do PURA) }\end{array}$ & Campinas, SP & 4,5 \\
\hline Nunes, 2000 & Campinas, SP & 134 \\
\hline $\begin{array}{c}\text { Nunes, } 2000 \\
\text { (após implantação do PURA) }\end{array}$ & Campinas, SP & 8 \\
\hline
\end{tabular}

Fonte: Soares (2016).

Gonçalves et al (2005) defendem que para a análise do consumo e do potencial de economia de água em uma edificação escolar, devam ser definidos outros indicadores além do indicador de consumo, como por exemplo, indicadores de perdas, e o índice de percepção dos usuários, que analisa o comportamento do usuário diante do uso racional da água.

Inserido nesse contexto, o presente trabalho contém estudos realizados em duas escolas estaduais da cidade de Recife-PE, objetivando o diagnóstico do padrão de consumo dessa tipologia predial. Este trabalho faz parte dos projetos, "Programa de conservação de água em escolas públicas" que é uma proposta de contribuição na temática da educação associada à 
conservação de água, através de ações educativas junto aos alunos, professores e funcionários de escolas públicas de Pernambuco, que poderão atuar como multiplicadores deste conhecimento no âmbito doméstico; e "Conservação de água em edificações públicas" que objetiva propor alternativas para atender à demanda de políticas de conservação e uso racional em áreas urbanas, em âmbito nacional, através de medidas viáveis para redução do consumo de água potável em escolas públicas e prédios públicos administrativos da Região Metropolitana do Recife. Ambos desenvolvidos pelo AquaPOLI - Grupo de Recursos Hídricos da Escola Politécnica de Pernambuco - POLI, na Universidade de Pernambuco.

\section{OBJETIVOS}

Diante do exposto, o presente estudo objetiva estabelecer indicadores de consumo de água para duas escolas piloto, uma de pequeno e a outra de médio porte, ambas pertencentes à rede pública estadual do município de Recife-PE, visando o diagnóstico preliminar do padrão de consumo dessa tipologia predial, através da medição setorizada do consumo de água nas duas escolas por um período de 01 (um) ano e do cálculo do indicador de consumo em litros/aluno/dia.

\section{METODOLOGIA}

A metodologia empregada consistiu, primeiramente, na seleção das duas escolas-piloto. Para tanto, em janeiro de 2014, um total de sete escolas foram previamente visitadas e cadastradas. A escolha das duas escolas-piloto levou em consideração a proximidade com o campus da Escola Politécnica da Universidade de Pernambuco para facilitar as visitas semanais, a receptividade da direção da escola e uma análise do histórico de consumo dos três anos anteriores.

Uma vez definidas as escolas, fez-se uma análise detalhada de todos os pontos de consumo de água, das atividades consumidoras e suas formas de realização através de entrevistas e observações em campo.

Através de parceira com a Companhia Pernambucana de Saneamento - Compesa, foram instalados hidrômetros em pontos estratégicos para o monitoramento setorizado do consumo de água entre os meses de fevereiro a abril de 2014. A leitura dos equipamentos foi feita numa frequência semanal, durante o ano de 2014.

A partir dos dados obtidos procedeu-se com a análise setorial de consumo. As escolas foram divididas em setores distintos, considerando o local no qual os hidrômetros estavam instalados, e os usuários característicos de cada ponto de consumo. Em seguida, foi calculado o consumo médio mensal de cada setor.

O Indicador de Consumo (IC) em litros/aluno/dia, foi calculado de acordo com a metodologia de Oliveira (1999), utilizando a seguinte equação:

$$
I C=\frac{C m \times 1000}{N A \times D m}
$$


Onde são considerados o consumo médio mensal $(\mathrm{Cm})$, o número de agentes consumidores (NA), e a quantidade de dias úteis por mês (Dm). Para o número de agentes consumidores (NA) foi adotado apenas o quantitativo de alunos, pois o Indicador de Consumo é uma referência de consumo em função de um agente específico, e em escolas a referência comumente utilizada é o número de alunos. Para a quantidade de dias úteis foi escolhido o número padrão de 22 dias por mês.

\section{RESULTADOS}

\subsection{SELEÇÃO DAS DUAS ESCOLAS PILOTO}

O Quadro 2 abaixo resume as sete escolas visitadas e cadastradas pela equipe do AquaPOLI, em janeiro de 2014.

Quadro 2: Dados das escolas cadastradas

\begin{tabular}{|l|c|c|c|c|c|}
\hline Amostra & Município & Bairro & $\begin{array}{c}\text { Consumo mensal } \\
\text { médio }\left(\mathbf{m}^{\mathbf{3}}\right)\end{array}$ & Desvio Padrão & 25\% da Média* \\
\hline Escola 01 & Recife & Cordeiro & 270,1 & 52,8 & 67,5 \\
\hline Escola 02 & Recife & Zumbi & 29,6 & 16,8 & 7,4 \\
\hline Escola 03 & Recife & Derby & 312,7 & 203,8 & 78,2 \\
\hline Escola 04 & Recife & Bongi & 95,5 & 13,4 & 23,9 \\
\hline Escola 05 & Recife & Boa Viagem & 142,5 & 54,7 & 35,6 \\
\hline Escola 06 & Camaragibe & Bairro Novo & 151,6 & 43,5 & 37,9 \\
\hline Escola 07 & Recife & Torre & 88,0 & 0,6 & 22,0 \\
\hline
\end{tabular}

*Média referente aos três últimos anos (2011, 2012 e 2013), excluindo os meses de janeiro, junho, julho e dezembro.

Fonte: Nunes (2015).

Para este trabalho foi adotado que a amostra deveria possuir um desvio padrão inferior a $25 \%$ da média do consumo dos últimos três anos, visto que um desvio padrão elevado pode indicar altos índices de perdas por vazamentos. Com isso, as escolas 01, 03, 05 e 06 foram consideradas inadequadas. A escola 07 não possuía hidrômetro nos anos de 2011 e 2012, o que poderia camuflar a veracidade dos dados, sendo então desclassificada. Consequentemente, as escolas 02 e 04 foram as selecionadas para serem as escolas piloto objeto desse estudo. A escola 04 corresponde à escola $\mathrm{A}$ e 02 à $\mathrm{B}$.

\subsection{CARACTERIZAÇÃO DAS ESCOLAS}

A Escola A possui uma área de $9.760 \mathrm{~m}^{2}$. Suas instalações hidrossanitárias são compostas de 18 torneiras convencionais e 12 bacias sanitárias, sendo 2 com caixa acoplada e com duplo acionamento, além de 4 chuveiros. Seu corpo discente é formado por alunos do ensino fundamental (6ㅇ ano ao 9o ano), ensino médio e educação de jovens e adultos, distribuídos nos três turnos. Em 2014, ano de realização da investigação em campo, a unidade possuía 
aproximadamente 1139 alunos, sendo 218 em horário integral e 72 funcionários, entre professores, coordenadores, monitores, vigias, cozinheiras e serviços gerais.

A Escola B contempla uma área de, aproximadamente, $1.560 \mathrm{~m}^{2}$. Suas instalações sanitárias totalizam 10 torneiras convencionais, 4 chuveiros ativos e 6 bacias sanitárias do tipo convencional com caixa de descarga não acoplada. O corpo discente, no ano de 2014, compreendia 394 alunos distribuídos em 12 turmas, do $5^{\circ}$ ao $9^{\circ}$ ano do ensino fundamental, incluindo duas turmas de Educação para Jovens e Adultos (EJA). O quadro de funcionários contava com um total de 33 profissionais ativos, entre professores, administrativos, serviçosgerais e merendeiras.

Enquanto a Escola $\mathrm{A}$ era abastecida apenas pela concessionária local, a Escola $\mathrm{B}$ recebia água da concessionária e de um poço, que passou a ser monitorado a partir da medição setorizada. Após o levantamento de todos os pontos de consumo de água das duas escolas, a Compesa cedeu e instalou 28 hidrômetros na Escola A e 15 na Escola B.

\subsection{CONSUMO POR SETOR}

A divisão do ambiente escolar em setores, de acordo com os pontos de consumo de água e o tipo de agente consumidor, é relevante para a caracterização do padrão de consumo das edificações em estudo, pois permite avaliar as áreas mais e menos consumidoras de água.

As escolas foram dividas em cinco setores distintos: funcionários, alunos-banheiros, alunossalas, copa e área comum. A descrição de cada setor encontra-se no Quadro 2 e os consumos mensais dos mesmos (em porcentagem), obtidos através dos dados coletados nos hidrômetros, são apresentados na Figura 1.

Quadro 2: Setores escolares para avaliação do consumo mensal

\begin{tabular}{|c|c|}
\hline Setor & Descrição \\
\hline Funcionários & $\begin{array}{l}\text { Pontos de água destinados ao corpo de funcionários da escola: professores, direção, } \\
\text { administrativo, serventes, seguranças, monitores, zeladores, copeiras, entre outros. }\end{array}$ \\
\hline Copa & $\begin{array}{l}\text { Pontos de água da copa da escola, onde são preparadas as refeições servidas aos } \\
\text { alunos. }\end{array}$ \\
\hline Alunos (banheiro) & Banheiros masculino e feminino destinados aos alunos. \\
\hline Alunos (salas) & Pontos de água em salas especiais, como laboratórios, sala de artes, etc. \\
\hline Área Comum & $\begin{array}{c}\text { Pontos de consumo que toda a comunidade escolar tem acesso, como bebedouro e } \\
\text { torneiras. }\end{array}$ \\
\hline
\end{tabular}


Figura 1: Consumo mensal de água por setor escolar (\%)

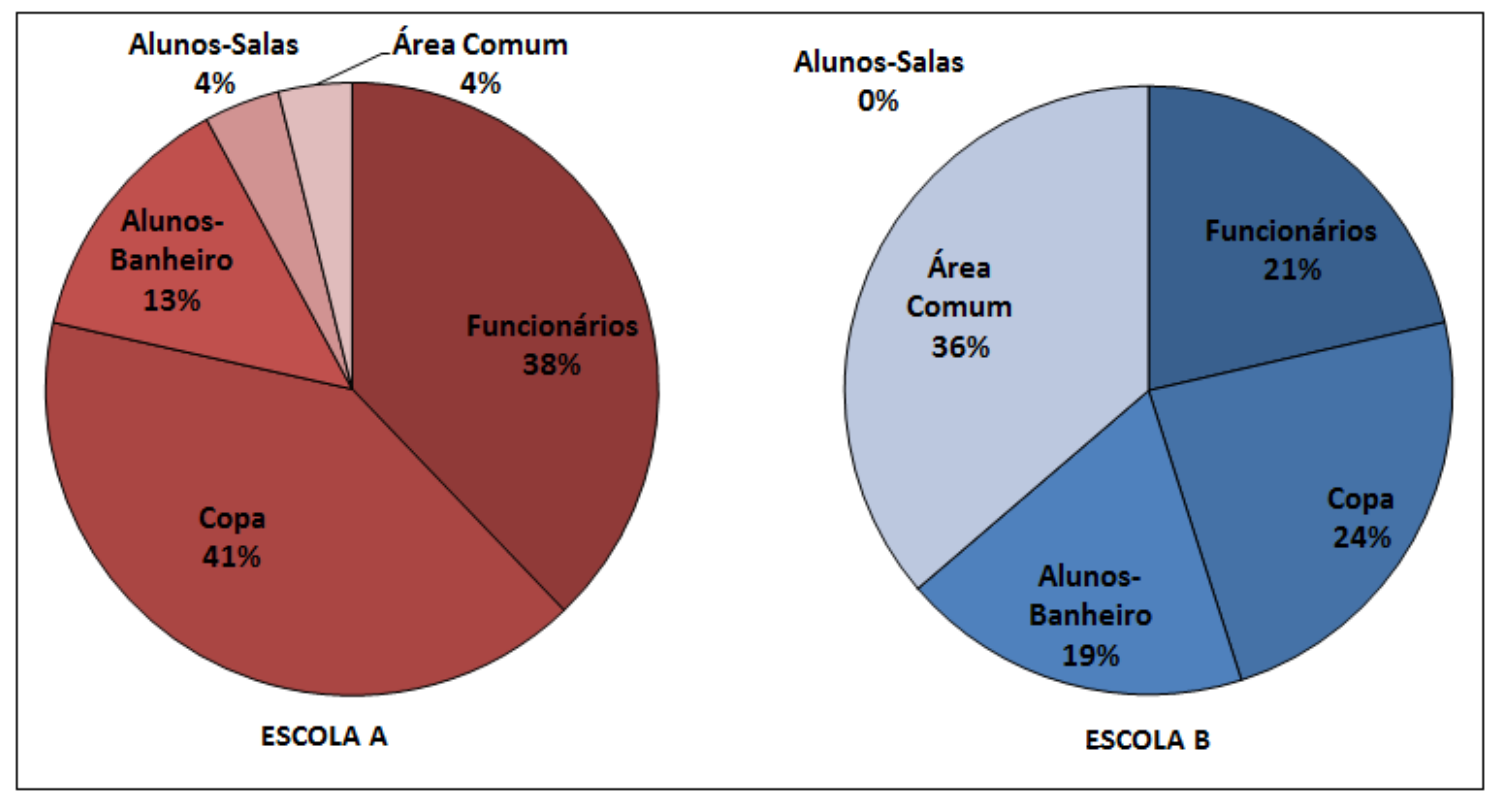

O setor de expressão no consumo mensal da água nas escolas é a copa. Nas duas instituições eram preparados três lanches e um almoço todos os dias. $O$ fato da escola $A$ ter um maior número de alunos, justifica que seu consumo nesse setor seja maior que na escola $B$, pois um número maior de refeições precisa ser preparado diariamente.

As duas escolas chamam a atenção pelo baixo consumo no banheiro dos alunos, principalmente a Escola $A$, cujo consumo médio mensal no referido setor chega a ser menor que em $B$, embora seu corpo discente seja 3,4 vezes maior. Observa-se também que nas duas escolas o consumo nos banheiros para uso dos funcionários, que representam a menor parcela de agentes consumidores, é maior que no banheiro dos alunos. Na escola $\mathrm{A}$ os funcionários correspondem a apenas $6 \%$ da população total, enquanto na escola B eles representam $8 \%$.

$A$ área comum, setor de maior consumo na escola $B$, compreende o bebedouro e duas torneiras utilizadas para lavar as salas e áreas externas na escola. Esse resultado pode ser devido ao fato de que o bebedouro fica localizado próximo das salas de aula, e os alunos podem facilmente ir beber água durante o intervalo entre uma aula e outra, tendo mais facilidade de acesso e uso do que os banheiros. As torneiras, por sua vez, são utilizadas para lavar as áreas externas e salas de aula, o que demanda uma grande quantidade de água. Em contrapartida, esse setor é o menos expressivo na escola A devido ao fato da frequência de limpeza das salas ser menor e da dificuldade dos alunos de acessarem o bebedouro em horário que não seja o de intervalo. Além disso, algumas salas na escola A possuíam pontos de água, compreendidos no setor "alunos-salas", fato que não ocorre na escola B.

De uma forma geral, fica evidente que os setores de uso exclusivamente dos funcionários apresentam um consumo superior ao dos alunos, que representam a massiva maioria da população escolar. 


\subsection{INDICADOR DE CONSUMO (IC)}

O Indicador de Consumo mensal (IC) para o ano de 2014 foi calculado de acordo com Oliveira (1999). Para tanto, foi estabelecida a quantidade de 22 dias úteis para todos os meses.

Tanto a escola A quanto a escola B apresentavam alunos que estudavam em dois turnos (horário integral), fator que precisou ser considerado na contabilização do número de agentes consumidores (NA). Nesse caso, quando o aluno ficava na escola todos os dias da semana, ele recebia peso 2. Outros alunos que ficavam em horário integral apenas 3 dias na semana contavam como 1,6 aluno.

O Gráfico 1 apresenta o IC mensal das escolas A e B. Os meses de junho, julho e dezembro não foram considerados por corresponderem a meses atípicos, com muitos recessos ou férias. Já janeiro, fevereiro e abril, foram os meses para a instalação completa de todos os hidrômetros, por isso também não foram considerados.

Gráfico 1: IC (L/aluno/dia) no ano de 2014

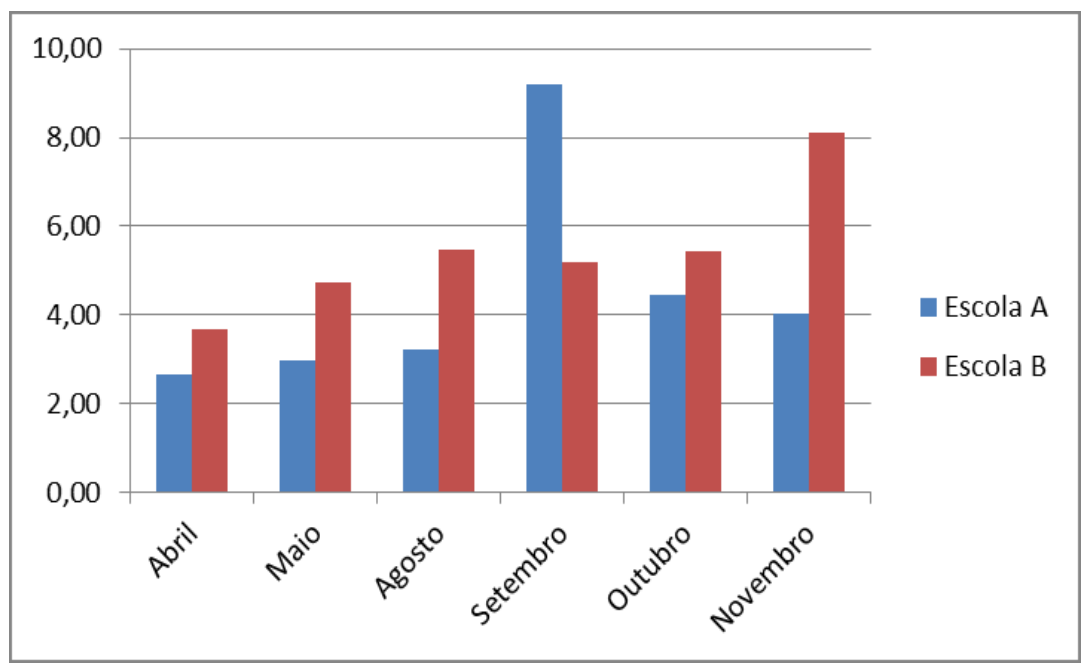

Para o ano de 2014, o indicador médio de consumo de água foi de 3,9 L/aluno/dia para a escola A (NA = 1270) e 4,9 L/aluno/dia para a escola B (NA = 428), que comparados com os diversos autores da literatura representam valores bem abaixo do esperado.

Para o projeto das instalações hidráulicas no Brasil, por exemplo, considera-se 50L/agente consumidor/dia em edificações escolares sob sistema de externato e 150L/agente consumidor/dia em escolas sob sistema de internato (Creder, 2006).

Gonçalves et al (2005) encontraram em Campinas, para escolas com as mesmas características das escolas objeto desse estudo, um indicador de consumo de água de 51,1 L/aluno/dia.

Oliveira (2013) estudou o indicador de consumo de 140 escolas das cidades que compõe a região denominada Triângulo Mineiro e verificou que 47,14\% das escolas investigadas apresentaram um consumo de água na faixa de 10,01 a 20,0 L/(agente consumidor.dia). Enquanto que escolas com faixa de consumo entre 0,0 a 10,0 L/(agente/consumidor.dia) representam $25,71 \%$ das escolas investigadas. 
Melo et al (2014) ressaltam que é possível estipular o consumo máximo de $10 \mathrm{~L} /$ (agente consumidor/dia) para escolas públicas após a instalação de equipamentos hidrossanitários econômicos ou implantação de Programa para Uso Racional da Água (PURA). No entanto, nem a escola A tão pouco a escola B se enquadram nessas situações.

Além disso, o valor de IC estabelecido para as duas escolas não considerou as perdas por vazamentos internos na rede, o que poderia reduzir ainda mais o IC encontrado. Gonçalves et al (2005) registraram perdas de até $74 \%$ para escolas de Campinas.

Portanto, esses baixos valores de IC encontrados nas escolas de Recife podem ser um reflexo do nível educacional ou social da população consumidora, como também podem representar um padrão do Estado. Porém, faz-se necessário o desenvolvimento de novos projetos em um número maior de escolas para verificar a ocorrência de casos semelhantes, ou não, e traçar melhor o perfil de consumo das escolas públicas do Recife.

\section{CONCLUSÕES}

Através da avaliação sistêmica dos usos da água no ambiente escolar, verificou-se que os setores de uso exclusivo da comunidade docente e funcionários de uma forma geral são os maiores consumidores de água, fato que parecia improvável tendo em vista que o quantitativo de alunos representa a maior parcela de usuários de água da escola ( $94 \%$ para a escola A e 92\% para a escola B). Essa realidade encontrada nas escolas evidencia a importância do diagnóstico preliminar do padrão de consumo na edificação para o desenvolvimento de ações visando à redução do consumo de água.

As escolas de Recife apresentaram um indicador de consumo bem abaixo dos 10L/aluno/dia, valor característico de outras escolas no Brasil onde foram implantados programas para uso racional de água (PURA). No entanto, nenhuma das escolas piloto apresentou equipamentos hidrossanitários com tecnologia economizadora ou programas de conscientização para o uso racional da água que justificassem os baixos valores de IC encontrados.

Como defendem diversos autores, o consumo de água pode ser influenciado por fatores regionais e socioeconômicos, o que poderia justificar essa diferença entre o Indicador de Consumo das escolas em estudo e o das outras escolas em diferentes regiões do Brasil.

Para trabalhos futuros, aconselha-se uma investigação no consumo de água dos alunos para identificar as razões deste ser tão baixo, podendo implicar em hábitos de higienização não adequados. Deve-se também investigar outros indicadores, como de vazamentos, perdas e percepção dos usuários, para tirar melhore conclusões quanto o potencial de economia das edificações.

A substituição dos equipamentos hidrossanitários convencionais para aqueles com tecnologia economizadora podem levar a uma redução ainda maior no consumo de água das escolas. Atrelado a isso, atividades de educação voltadas a conservação de água devem ser desenvolvidas a fim de criar uma cultura de responsabilidade social e ambiental nos alunos e em todos os profissionais que compõe o quadro da escola.

A análise desses indicadores deve orientar o desenvolvimento de atividades para sensibilização dos usuários direcionadas para cada setor específico, contribuindo para a redução do 
desperdício de água. O envolvimento de toda a população da escola (alunos, professores, administração e demais funcionários) é indispensável para que o emprego da água seja mais eficiente no ambiente escolar. Para tanto, os diversos usuários precisam estar cientes de como influenciam no consumo e como podem contribuir no processo de redução do mesmo.

\section{REFERÊNCIAS}

AGÊNCIA NACIONAL DE ÁGUAS (ANA). Conjuntura dos Recursos Hídricos no Brasil. Brasília, DF. 2013.

CHENG, C. L.; HONG, Y. T.; Evaluating water utilization in primary schools. Building and Environment, v. 39, p. 837-845. 2004.

CHEUNG, P. B.; KIPERSTOK, A.; COHIM, E.; ALVES, W.C.; PHILIPPI, L.S.; ZANELLA, L.; ABEN.; GOMES, H.P.; SILVA, B.C.; PERTEL, M.; GONÇALVES, R.F. Consumo de água. In: GONÇALVES, R. F.(Coord.). Uso Racional de Água e Energia: conservação de água e energia em sistemas prediais de abastecimento de água. Vitória: ABES-PROSAB, 350 p., 2009.

CREDER, Hélio. Instalações Hidráulicas e Sanitárias. 6.ed. Rio de Janeiro. Ed. LTC - Livros Técnicos e Científicos, 2006. 465 páginas.

FARINA, M.; MAGLIONICO, M.; POLLASTRI, M.; STOJKOV, I.; Water consumptions in public schools. Procedia Engineering, v.21, p. 929 - 938. 2011.

GONÇALVES, O.; ILHA, M.; AMORIM, S.; PEDROSO, L. Indicadores de uso racional de água para escolas de ensino fundamental e médio. Ambiente Construído, Porto Alegre, v.5, n.3, p.35-48, jul/set, 2005.

MELO, N. A.; SALLA, M. R.; OLIVEIRA, F. R. G. de; FRASSON, V. M. Consumo de água e percepção dos usuários sobre o uso racional de água em escolas estaduais do triângulo mineiro. Ciência \& Engenharia, jul-dez, 2014.

NUNES, L. G. C. F. Indicadores de Consumo de Água, em uma Escola Estadual de Recife-PE. Monografia de Bacharel em Engenharia Civil. Escola Politécnica da Universidade de Pernambuco. Recife, PE. 2015.

NUNES, S. da S. Estudo da conservação de água em edifícios localizados no Campus da Universidade Estadual de Campinas. Dissertação de Mestrado em Engenharia Civil. Universidade Estadual de Campinas. Campinas, SP. 2000.

OLIVEIRA, L. H. Metodologia para a implantação de programa de uso racional de água em edifícios. Tese de doutorado em Engenharia Civil - POLI, Universidade de São Paulo. São Paulo, 1999.

OLIVEIRA, F. R. G. de. Consumo de água e percepção dos usuários para o uso racional da água em escolas estaduais de Minas Gerais. Dissertação de Mestrado. Universidade Federal de Uberlândia. Uberlândia, 2013.

Programa Nacional de Combate ao Desperdício de Água (PNCDA). Documento Técnico de Apoio no A5: Planos Regionais e Locais de Combate ao Desperdício de Água - Diretrizes. Brasília, DF. 2003

REBOUÇAS, A. C. Água na Região Nordeste: desperdício e escassez. Estudos Avançados, USP 11 (29), p 127 - 154. 1997.

REBOUÇAS, A. C. Água e desenvolvimento rural. Estudos Avançados, USP 15 (43), p 327 - 3444.2001. 
REFERENCIAL técnico de certificação "Edifícios do setor de serviços - PROCESSO AQUA" Escritório e Edifícios escolares. FCAV, out/2007. Versão 0.

SOARES, A. E. P. Análise do Consumo de Água em uma Escola Pública Estadual de Recife-PE. Monografia de Bacharel em Engenharia Civil. Escola Politécnica da Universidade de Pernambuco. Recife, PE. 2016. 